\title{
Clusterization, frustration and collectivity in random networks
}

\author{
Anna Mańka, Krzysztof Malarz and Krzysztof Kułakowski* \\ Faculty of Physics and Applied Computer Science, \\ AGH University of Science and Technology, \\ al. Mickiewicza 30, PL-30059 Kraków, Euroland \\ *kulakowski@novell.ftj.agh.edu.pl
}

August 14, 2018

\begin{abstract}
We consider the random Erdős-Rényi network with enhanced clusterization and Ising spins $s= \pm 1$ at the network nodes. Mutually linked spins interact with energy $J$. Magnetic properties of the system as dependent on the clustering coefficient $C$ are investigated with the Monte Carlo heat bath algorithm. For $J>0$ the Curie temperature $T_{c}$ increases from 3.9 to 5.5 when $C$ increases from almost zero to 0.18 . These results deviate only slightly from the mean field theory. For $J<0$ the spin-glass phase appears below $T_{S G}$; this temperature decreases with $C$, on the contrary to the mean field calculations. The results are interpreted in terms of social systems.
\end{abstract}

PACS numbers: 89.65.-s, 64.90.+b

Keywords: random networks; phase transitions;

\section{Introduction}

Research on systems with random topology was initialized by Paul Flory in 1941 [1, 2], but they reappeared only about nine years ago [3] as a subject of great interdisciplinary interest with applications in many sciences, from transport to biology [4, 5, 6]. Most of effort is concentrated on the topological structure of growing networks, where the scale-free degree distribution has been found. However, progress is achieved in the science of networks as a whole.

In particular, networks provide a convenient basis of modelling social systems [7. However, the structure of social networks remains debatable. As it was discussed by Michael Schnegg 9, the actual topology of a social network depends on social relations in the society. Schnegg investigated six small-scale societies, mostly African ones. The degree distribution of three of them were found to be close to decreasing functions with some fluctuations, whereas the other three displayed a maximum. As the determinant what allows to differ between these two groups, Schnegg proposed the amount of reciprocity; when it is large, the model degree distribution is close to the Poisson function, known to appear in the classical Erdős-Rényi random networks (4, 8. When the reciprocity is small, the system is supposed to be close to a scale-free network 9]. It seems that the topology of a social structure varies from one society to another, and various kinds of networks can be appropriate in different cases. We note only that, as remarked in Ref. [10, the clustering coefficient in the social networks is much larger, than in the Erdős-Rényi networks. 
On the other hand, the topology itself does not reflect the richness of behaviour of the social systems, and it is worthwhile to develop a theoretical description of these systems with more degrees of freedom. The simplest method is to decorate the network nodes $i=1, \ldots, N$ with additional variables, as Ising spins $s_{i}= \pm 1$. These variables are not statistically independent. The system total energy is given as $E=-J / 2 \sum_{i j} s_{i} s_{j}$, where the summation goes via all connected pairs of nodes/spins and $J$ is the so-called exchange integral. Two basic cases: ferromagnetic interaction $(J>0)$ which prefers the same sign of neighboring spins, and antiferromagnetic interaction $(J<0)$, which prefers their opposite signs, are to be discussed separately; for a recent review see [11. In each case, the interaction competed with some noise, commonly modeled as the thermal noise. In numerous cases, a variation of the noise intensity leads to a phase transition, from the phase with ordered spins (low noise) to the phase of disordered spins (large noise). The amount of noise where the ordered phase disappears is comparable to the Curie temperature in ferromagnets. In fact, spin degrees of freedom ruled by some stochastic evolution has been used many times to describe social processes, as for example the opinion dynamics 12. In these works, ordering of spins can be treated as a demonstration of a collective behaviour despite of the presence of noise. The noise level is measured by parameter $T$ and usually called temperature. Then the probability of move in the system states space from state ' 1 ' of energy $E_{1}$ to the state '2' of energy $E_{2}$ may be given by the probability $\left.p_{1 \rightarrow 2}=[1+\exp (2 \Delta E / T))\right]^{-1}$, where $\Delta E=E_{2}-E_{1}$. Such dynamic rule is termed as the heat-bath algorithm [13]. When we deal with the social systems, a society can be modeled by the network and the Curie temperature $\left(T_{c}\right)$ can be treated as a measure of an ability of a society to a collective action. This ability is expected to depend on the topology of interpersonal bonds. In terms of magnetism, the Curie temperature depends on the topology of the investigated network [14.

The case of antiferromagnetic interaction is of special interest for sociophysics. As it was argued only recently [15], a dichotomous behaviour is found in some circumstances, as buying or selling [16]. Another example is the dove or hawk strategy [17. In this case the topology of the social network is particularly important because of the geometrical frustration effect, which removes the unique and deep minimum of the magnetic energy. Instead, numerous local stable or metastable states appear, and the structure of the set of these states remains unsolved [18. We note in passing that the dynamics of the magnetic behaviour of antiferromagnetic growing networks was found to be surprisingly rich 19 .

Here we are interested in the Erdős-Rényi random network decorated with Ising spins, with ferromagnetic or antiferromagnetic interaction. To enhance the clusterization coefficient, we apply the algorithm proposed by Holme and Kim in Ref. 20] for the growing networks; it is easy to generalize it for the random networks. Other methods of network design have been proposed for example in Ref. 21]. Our aim is to investigate, how the clustering influences the system behaviour.

The Curie temperature for the random network was obtained already by Sumour et al. 22. However, up to our knowledge the clusterization coefficient has not been varied in these networks. Recently, similar problem for $J>0$ was also considered 23 in the hierarchical network, designed by Watts et al. 24]. In that network, the clustering was controlled by the probability of linking 
different clusters. Varying it, one moved from the random network to a set of separate clusters. The obtained Curie temperature varied from $3.8 J$ to zero in a step-like way, very sharply. Owing to algorithmic/numerical peculiarities of the hierarchical network, the size of the system investigated in [23] was limited. Also, the clustering led the system to split into subnetworks, what does not occur in our case. In the antiferromagnetic case $J<0$, the uncorrelated network is believed to show the spin-glass phase at low temperature 27, 28. As all $J$ 's are negative, there is no bond disorder; the frustration is introduced by the disorder of the network structure and it is purely geometrical [29].

\section{The results}

At the first stage of the simulation, a random Erdős-Rényi network is constructed. The starting point is a set of $N=10^{6}$ nodes. A link is placed between each two nodes with the probability $p$. Next, the clustering is enhanced according to the receipt in Ref. 20]: nodes are selected with two or more neighbours, and a new link is placed between each pair of these neighbours with probability $r$ selected as to get the average degree $\langle k\rangle=4$.

The clusterization coefficient $C$ is defined as the average over nodes $i=$ $1, \ldots, N$ of the local coefficient $C_{i}$, where

$$
C_{i}=\frac{2 y_{i}}{k_{i}\left(k_{i}-1\right)}
$$

$k_{i}$ is the degree of $i$-th node $i . e$. the number of nodes linked to $i$, and $y_{i}$ is the actual number of links between these $k_{i}$ nodes. The maximal value of $C_{i}$ is one. In our system, the coefficient $C$ varies from almost zero (when $p=4 / N$ and $r=0)$ to 0.18 . This enhancement appears to influence the degree distribution. As it is shown in Fig. 1, the plot is Poissonian for small $C$, but deviates from this curve for $C=0.18$.

The magnetic behaviour of the system is also influenced by the clusterization. Let us start from the ferromagnetic case, where the exchange integral $J$ is set to +1 . We calculate the average magnetization $M=\sum_{i} s_{i} / N$ against temperature, $M(T)$, for $r=0$. Initially, all spins are set to +1 , and the time average is taken after $N_{t}$ time steps from another $N_{t}$ time steps. One timestep is equivalent to $N$ attempts to flip a spin. For the comparison with the results of 22 , the calculations for $\langle k\rangle=4$ are repeated for $\langle k\rangle=3$ and $\langle k\rangle=2, N=10^{4}$. The obtained curves for the squared magnetization are presented in Fig. 2. As we see, the obtained Curie temperature increases with $\langle k\rangle$. Also, the mean-fieldcharacter of the system is confirmed, as it was concluded in [22], because the curves $M^{2}(T)$ are approximately linear. For $\langle k\rangle=2$, the value of the Curie temperature, $T_{c} / J \approx 1.7$ agrees with the one obtained in $[22$. For $\langle k\rangle=4$, the value of the Curie temperature $T_{c} / J \approx 3.8$ agrees with the result of [23] for the case of random network, where the homophily parameter $\alpha=-\ln 2$

(for the discussion of the interpretation of $\alpha$ see Ref. 24]). We note also that the analytical solution 14 for the uncorrelated random networks with the Poissonian degree distribution

$$
\frac{J}{T_{c}}=\frac{1}{2} \ln \left(\frac{\left\langle k^{2}\right\rangle}{\left\langle k^{2}\right\rangle-2\langle k\rangle}\right)
$$




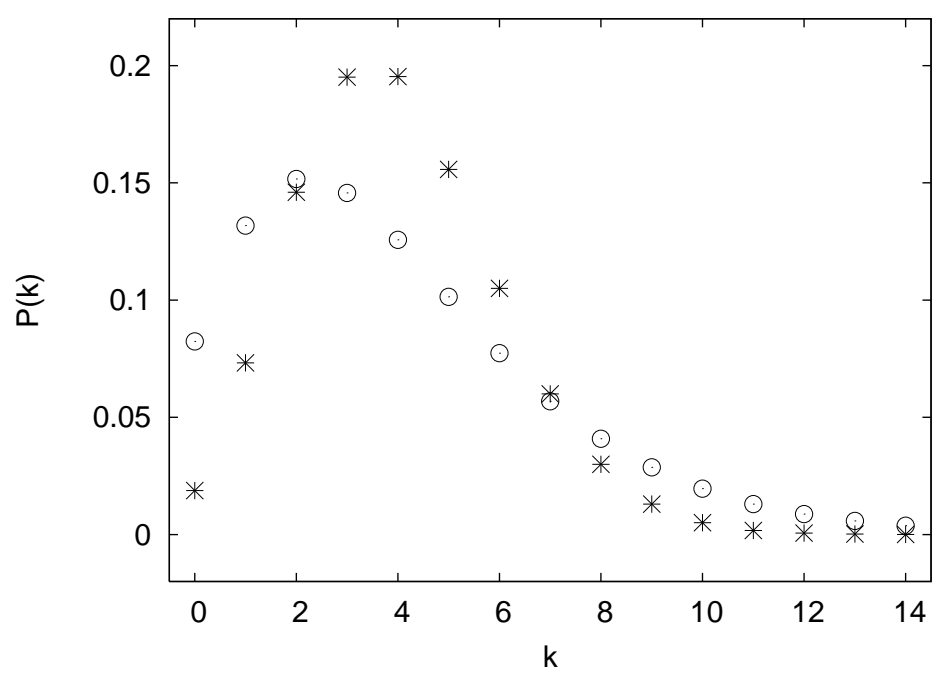

Figure 1: The degree distribution for the random network with $C \approx 0$ (stars) and $C=0.18$ (circles), for $N=10^{6}$ nodes. The former is the Poisson distribution for $\langle k\rangle=4$ and $C \approx 0$; there, $\left\langle k^{2}\right\rangle=20.0$ and $z_{2}=16.0$. For $C=0.18$ the simulation gives $\left\langle k^{2}\right\rangle=25.5$ and $z_{2}=21.5$.

gives $T_{c} / J=3.91,2.88$ and 1.82 for $\langle k\rangle=4,3$ and 2 , respectively. The obtained values are slightly smaller. The differences are due mainly to the finite-size effect.

These calculations were repeated again for $\langle k\rangle=4, J>0, r>0$ and a larger network, i.e. $N=10^{5}$. The results shown in Fig. 3 indicate, that the Curie temperature increases with the clustering coefficient $C$. This increase does follow from the increase of the second moment $\left\langle k^{2}\right\rangle$ of the degree distribution. As shown in Fig. [4 the theoretical estimation of Eq. (2) differs only slightly from the observed value of $T_{c}$. The same is true for the more general formula 11

$$
\frac{J}{T_{c}}=\frac{1}{2} \ln \left(\frac{z_{2}+z_{1}}{z_{2}-z_{1}}\right)
$$

where $z_{1}=\langle k\rangle=4$, and $z_{2}$ is the number of second neighbours. Both equations 2 and 3 give the same theoretical values. Indeed, our numerical calculations confirm the relation $z_{2}=<k(k-1)>$.

In the antiferromagnetic case $(J<0)$ the disordered topology of the random network does not allow to investigate the staggered magnetization as the order parameter. We made an attempt to calculate the magnetic specific heat $C_{V}$ against temperature. For the lattice antiferromagnets, it is the maximum of $C_{V}$ at given temperature what marks an existence of the phase transition. Indeed, we found an indication of such a transition for the case $r=0$, where the clusterization coefficient vanishes. Slightly below the maximum, $C_{V}$ obtained from the derivative of energy with respect to $T$ starts to differ from $C_{V}$ calculated from the variance of energy. This split is due to the lack of thermal equilibrium 


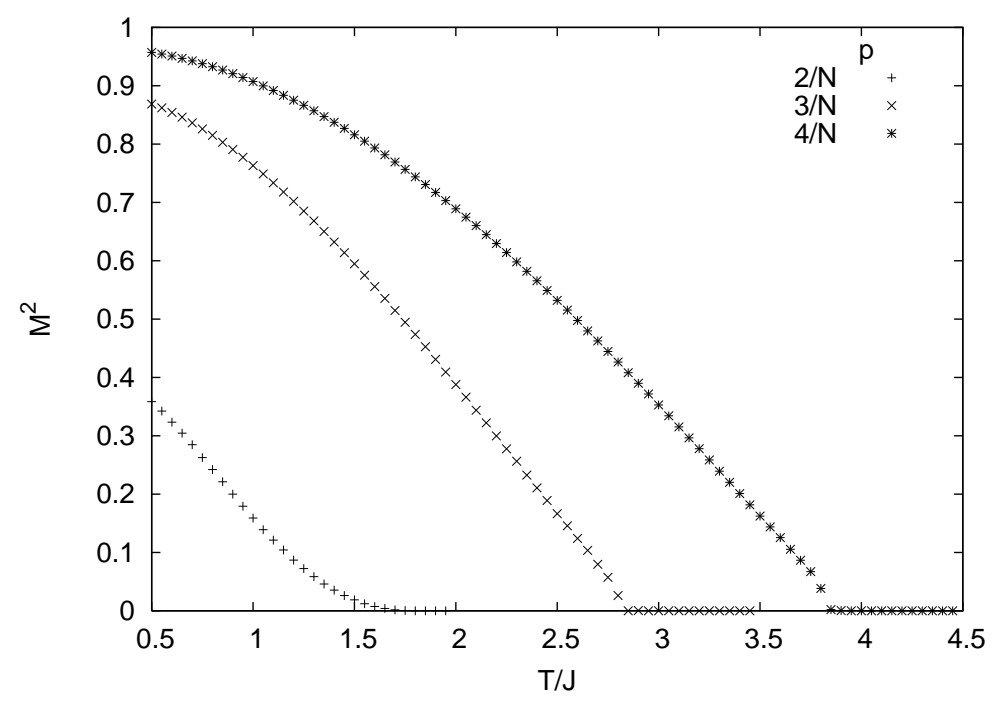

Figure 2: Thermal dependence of the squared magnetization for $J>0, N=10^{4}$ nodes, $r=0$. The mean node degree $\langle k\rangle$ is 2 (left curve), 3 (middle) and 4 (right curve). $\left(N_{\text {iter }}=2 \cdot 10^{4}\right)$.

in the spin-glass phase. Still, the position of the maximum can be determined. In Fig. 5, only the result from the thermal derivative of energy is shown. These calculations are preceded by $N_{t}=10^{4}$ time steps, till the time dependence of energy reaches the plateau; this was checked to be long enough if $T>0.7$.

The plots shown in Fig. 5 show that the transition temperature $T_{S G}$ decreases with the clustering coefficient $C$. This result is confirmed by the calculations of the Edwards-Anderson spin-glass order parameter $q$ [25, 26]

$$
q=\frac{1}{N} \sum_{i}\left(\frac{1}{\tau} \sum_{t=1}^{\tau} s_{i}(t)\right)^{2}
$$

where $s_{i}(t)$ is $i$-th spin at time $t$. The obtained plots for $q(T)$ for various $C$ are shown in Fig. 6. Here also, the temperature where $q$ vanishes decreases from 1.9 for $C=0$ to about 1.4 for larger values of $C$.

\section{Discussion}

New results obtained for the ferromagnetic case indicate that the enhancement of the clusterization by additional links does not invalidate the assumption of the lack of correlations, which is usually made in the theory [14 of random networks. However, for $C=0.14$ and above this value, the loops introduced to the system slightly enhance the Curie temperature, as shown in Fig. 4.

For the antiferromagnetic case, the situation is more complex. For negligible clusterization, the maximum of the specific heat indicates the phase transition between the paramagnetic and the spin-glass phase. The transition temperature $T_{S G}$ rougly accords with the mean field result $T_{S G}=1.91$ from Eq.5. 


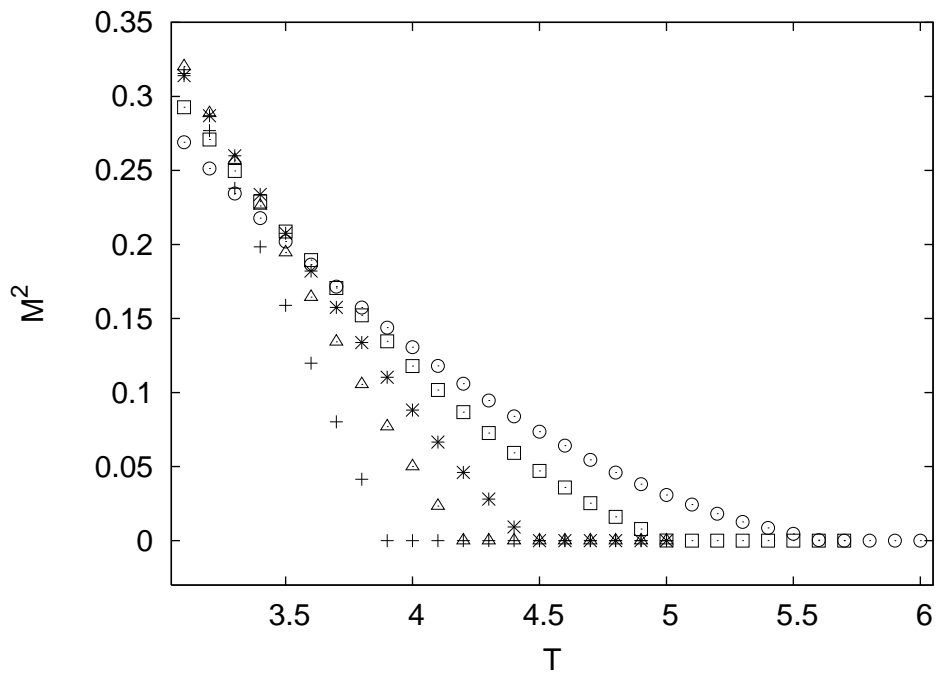

Figure 3: Thermal dependence of the squared magnetization for $J>0$, different values of $C$, and $N=10^{5}$ nodes. The mean node degree $\langle k\rangle=4$. The clustering coefficient $C \approx 0,0.05,0.09,0.14$ and 0.18 from left to right.

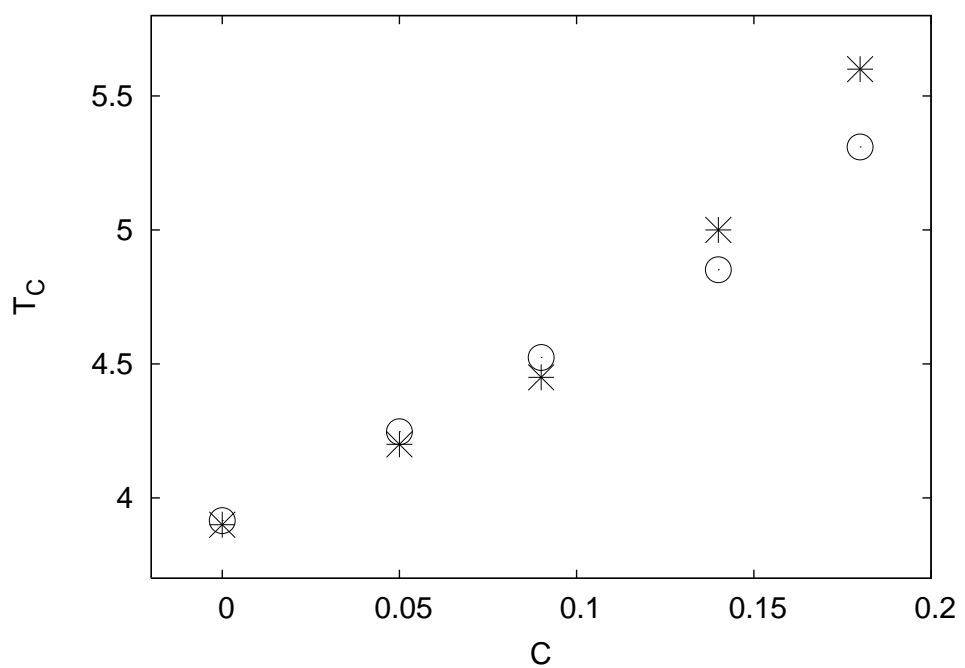

Figure 4: The Curie temperature obtained from the simulation (crosses) and from Eq. (2) or (3) (circles). 


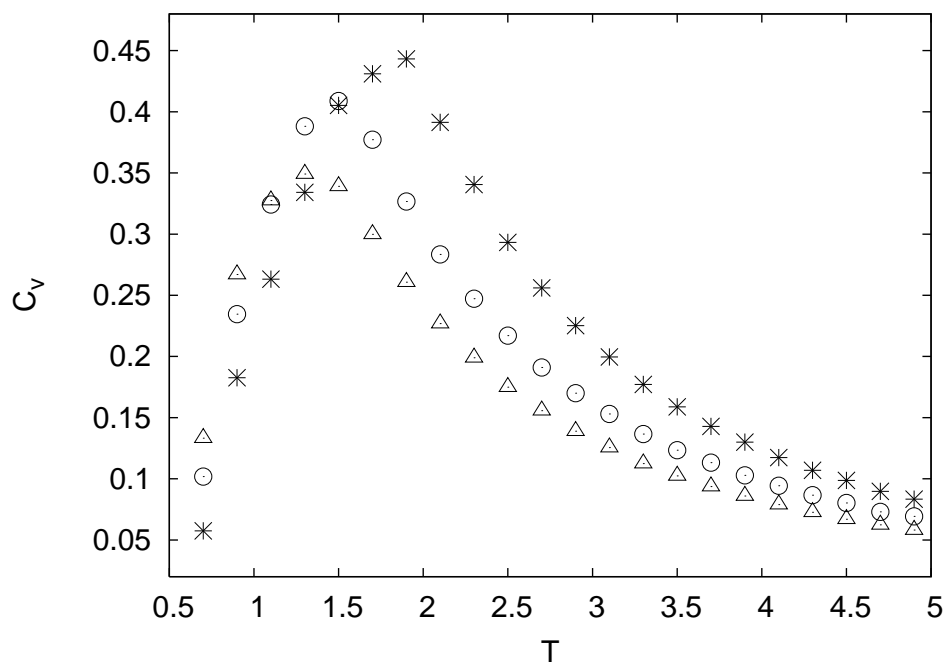

Figure 5: The magnetic specific heat $C_{V}(T)$ for the case $J<0$, where $C=0$, 0.09 and 0.18 (crosses, circles and triangles, respectively).

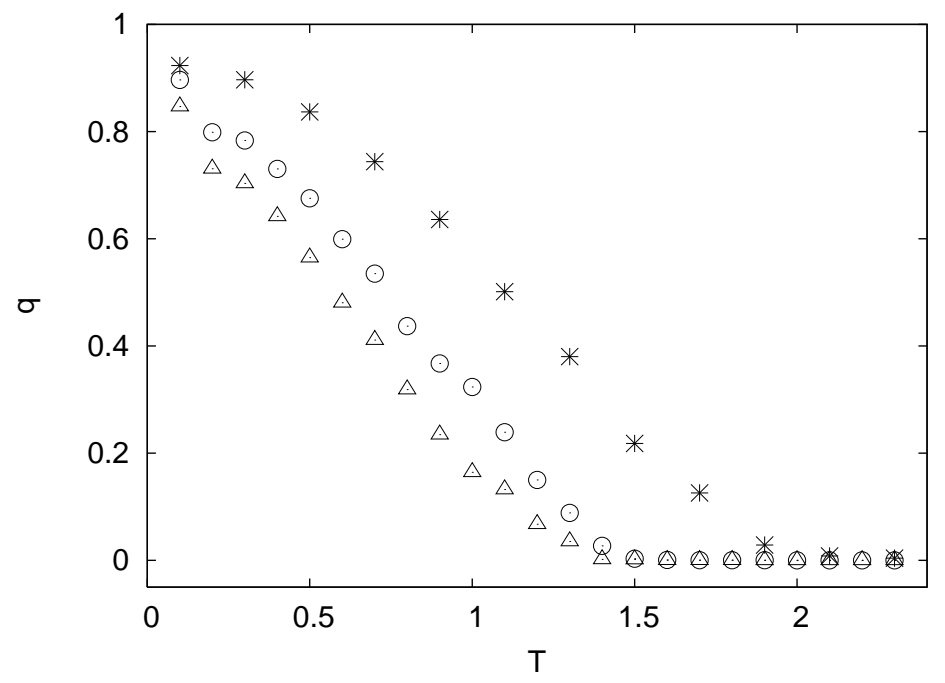

Figure 6: The Edwards-Anderson order parameter $q(T)$ for the case $J<0$, where $C=0,0.09$ and 0.18 (crosses, circles and triangles, respectively). 


$$
\frac{z_{1}}{z_{2}}=\tanh ^{2}\left(\frac{1}{T_{S G}}\right)
$$

which follows from Eq. 92 in Ref. [11 and from our delta-like distribution $\rho(J)=\delta(J+1)$ of the exchange integral $J$. However, the theoretical values of $T_{S G}$ increase with $C$ to 1.92 for $C=0.09$ and to 2.17 for $C=0.18$. Our numerical results on $C_{V}$ and $q$ indicate, that actually $T_{S G}$ decreases with $C$.

The topology of the system allows to treat it as a disordered version of the Archimedean lattice, discussed in Ref. 26. There, the triangles of spins were connected as to form the periodic structure. The ground state degeneracy of this Archimedean lattice increases exponentially with the system size. The same rule applies to our disordered lattice if the density of the triangles is large enough.

As discussed in Ref. [11, there are links from the problem of spin-glass in disordered networks to some interested NP-complete problems as MAX-CUT or the satisfiability. It seems that some counterparts of our system can be found also in the game theory, and in particular in the network congestion games [30]; for a recent review of games on networks see Ref. 31. To maintain our social interpretation, we can state that the case of a ferromagnet the smooth increase of the Curie temperature with the coefficient $C$ marks the fact that the ordered phase is less fragile if the system has the clustered structure. Considering an antiferromagnet we take into account that effects of social interactions are sometimes incoherent, that the society can be polarized in some way and that it is possible that increasing the density of social ties can be in conflict with an ability to find best solutions to everyday problems. In this sense, geometrical frustration combined with noise can reproduce some social phenomena which disable a coherent social action. A next step to reflect features of the society should be to consider a distribution of positive and negative interaction of different sizes. This, however, requires much larger conceptual and numerical effort.

\section{Acknowledgements}

The authors are grateful to Dietrich Stauffer for helpful suggestions and critics. The calculations were performed in the ACK Cyfronet, Cracow, grants No. MNiSW /SGI3700 /AGH /030/ 2007 and MNiSW /IBM BC HS21 /AGH /030 $/ 2007$.

\section{References}

[1] P. Flory, Am. J. Phys. 63 (1941) 3083.

[2] D. Stauffer, arXiv:0704.3849.

[3] D. J. Watts and S. H. Strogatz, Nature 393 (1998) 440.

[4] R. Albert and A.-L. Barabási, Rev. Mod. Phys. 286 (2002) 47.

[5] S. N. Dorogovtsev and J. F. F. Mendes, Adv. Phys. 51 (2002) 1079. 
[6] M. E. J. Newman, SIAM Rev. 45 (2003) 167.

[7] D. J. Watts, Annu. Rev. Sociol. 30 (2004) 243.

[8] B. Bollobás, Random Graphs, Cambridge UP, Cambridge 2001.

[9] M. Schnegg, Int. J. Mod. Phys. C 17 (2006) 1067.

[10] M. E. J. Newman, Phys. Rev. E 64 (2001) 046132.

[11] S. N. Dorogovtsev, A. V. Goltsev and J. F. F. Mendes, $\operatorname{arXiv:0705.0010}$

[12] F. Schweitzer and J. Hołyst, Eur. Phys. J. B 15 (2000) 723.

[13] C. Michael, Phys. Rev. B 33 (1986) 7861.

[14] S. N. Dorogovtsev, A. V. Goltsev and J. F. F. Mendes, Phys. Rev. E 66 (2002) 016104.

[15] G. Weisbuch and D. Stauffer, Physica A 384 (2007) 542.

[16] D. Challet, M. Marsili and Y.-C. Zhang, Minority Games, Oxford UP, Oxford 2004.

[17] P. D. Straffin, Game Theory and Strategy, Math. Assoc. of America, Washington 1983.

[18] K. Binder and A. P. Young, Rev. Mod. Phys. 58 (1986) 801.

[19] K. Malarz, W. Antosiewicz, J. Karpińska, K. Kułakowski and B. Tadić, Physica A 373 (2007) 785.

[20] P. Holme and B. J. Kim, Phys. Rev. E 65 (2002) 026107.

[21] M. A. Serrano and M. Boguna, Phys. Rev. E bf 74 (2006) 056115.

[22] M. A. Sumour, A. H. El-Astal, F. W. S. Lima, M. M. Shabat and H. M. Khalil, Int. J. Mod. Phys. C 18, 53 (2007).

[23] W. Wołoszyn, D. Stauffer and K. Kułakowski, Eur. Phys. J. B, 57 (2007) 331.

[24] D. J. Watts, P. S. Dodds and M. E. J. Newman, Science 296 (2002) 1303.

[25] S. F. Edwards and P. W. Anderson, J. Phys. F: Met. Phys. 5 (1975) 965.

[26] M. J. Krawczyk, K. Malarz, B. Kawecka-Magiera, A. Z. Maksymowicz and K. Kułakowski, Phys. Rev. B 72 (2005) 024445.

[27] T. Castellani, F. Krzakala and F. Ricci-Tersenghi, Eur. Phys. J. B 47 (2005) 99.

[28] M. O. Hase, J. R. L. de Almeida and S. R. Salinas, submitted to J. Phys. A (arXiv: cond-mat/0604144).

[29] J. F. Sadoc and R. Mosseri, Geometrical Frustration, Cambridge UP, Cambridge 1999. 
[30] B. Vöcking, Congestion Games: Optimization in Competition, in Proc. 2nd ACiD (Durham, 2006), 9-20.

[31] G. Szabó G. Fáth, Physics Reports 446 (2007) 97. 